Pure Mathematical Sciences, Vol. 2, 2013, no. 1, 23 - 28

HIKARI Ltd, www.m-hikari.com

\title{
A Note on the Order Bidual of f-Algebras
}

\author{
Esra Uluocak* and Ömer Gök** \\ * Istanbul Arel University, Faculty of Science and Letters \\ Department of Mathematics and Computer Science, Turkey \\ esraaltinbilezik@arel.edu.tr \\ ** Y1ldız Technical University, Faculty of Arts and Science \\ Department of Mathematics, Turkey \\ gok@yildiz.edu.tr
}

Copyright (C) 2013 Esra Uluocak and Ömer Gök. This is an open access article distributed under the Creative Commons Attribution License, which permits unrestricted use, distribution, and reproduction in any medium, provided the original work is properly cited.

\begin{abstract}
The paper deals with the Arens Multiplication which we accomplished in four steps in the order bidual $X^{\sim \sim}$. It is shown that if $f$ is an element of order dual $X^{\sim}$ of $X$ with $\varepsilon(f) \neq 0$ and $x \in X^{+}$, then $f . x=0$ implies $f(x)=0$.

Mathematics Subject Classification : 06F25, 47B65
\end{abstract}

Keywords: Arens multiplication, f-algebra, Dedekind complete, orthomorphism, order dual.

\section{Introduction}

A Riesz space $E$ under an associative multiplication is said to be a Riesz algebra whenever the multiplication makes $E$ an algebra (with the usual properties ), and in addition it satisfies the following property : If $x, y \in E^{+}$, then $x y \in E^{+}$. A Riesz algebra $E$ is said to be an $f-a \lg e b r a$ whenever $x \wedge y=0$ implies $(x z) \wedge y=0$ for each $z \in E^{+}$. An order bounded band preserving operator is known as an orthomorphism and the set of all orthomorphism on $X$ is denoted by $\operatorname{Orth}(X)$, [2] . 
A subset $A$ of Riesz space is said to be bounded from above whenever there exists some $x$ satisfying $y \leq x$ for all $y \in A$. Similarly, a set $A$ is said to be bounded from below whenever there exists some $x$ such that $x \leq y$ holds for all $y \in A$. Finally, a set $A$ is called order bounded if it is bounded both from above and below. An operator $T: E \rightarrow F$ that maps order bounded subsets of $E$ onto order bounded subsets of $F$ is called order bounded. An operator $T: E \rightarrow E$ on a Riesz space is said to be band preserving whenever $T$ leaves all bands of $E$ invariant, i.e., whenever $T(B) \subseteq B$ holds for each band $B$ of $E$.

Let $\mathrm{E}$ be a Riesz space. A linear functional $f: E \rightarrow R$ is called order bounded if $f$ maps order bounded subsets of $E$ onto bounded subsets of $R$, [4]. The vector space $E^{\sim}$ of all order bounded linear functionals on $E$ is called the order dual of $E$, i.e., $E^{\sim}:=L_{b}(E, R)$. Let $X$ be an Archimedean $f-$ algebra with order dual $X^{\sim}$. Following construction $[3,5,6,7]$ a multiplication can be introduced in the order bidual $X^{\sim \sim}$ of $X$.

This is accomplished in four steps as explained below.

1) $\operatorname{Orth}(X) \times X \rightarrow X$

$$
(T, x) \rightarrow T \cdot x=T(x) \quad \text { for } \quad T \in \operatorname{Orth}(X), x \in X
$$

2) $X \times X^{\sim} \rightarrow \operatorname{Orth}(X)^{\sim}$

$$
\left(x, x^{\prime}\right) \rightarrow\left(x . x^{\prime}\right) T=x^{\prime}(T x) \quad \text { for } \quad x^{\prime} \in X^{\sim}, x \in X, T \in \operatorname{Orth}(X) .
$$

3) $\operatorname{Orth}(X)^{\sim} \times X^{\sim} \rightarrow X^{\sim}$

$$
\left(T, x^{\prime}\right) \rightarrow\left(T \cdot x^{\prime}\right) x=T\left(x . x^{\prime}\right) \quad \text { for } T \in \operatorname{Orth}(X)^{\sim}, x^{\prime} \in X^{\sim}, x \in X
$$

4) $\operatorname{Orth}(X)^{\sim} \times X^{\sim \sim} \rightarrow X^{\sim \sim}$

$$
(T, \hat{x}) \rightarrow(T \cdot \hat{x})\left(x^{\prime}\right)=\hat{x}\left(T \cdot x^{\prime}\right) \quad \text { for } T \in \operatorname{Orth}(X)^{\sim}, x^{\prime} \in X^{\sim \sim}
$$

Proposition 1 :Let $\alpha: \operatorname{Orth}(X)^{\sim} \rightarrow \operatorname{Orth}\left(X^{\sim}\right) \quad$ be a mapping defined by $\alpha(T) x^{\prime}=T x^{\prime}$ for $T \in \operatorname{Orth}(X)^{\sim}, \quad x^{\prime} \in X^{\sim}$. Then $\alpha$ is a one-one, onto and algebra homomorphism.

Proof : We will prove the following, respectively. 
i) $\alpha$ is a linear mapping.

ii) $\alpha$ is an one-one mapping.

iii) $\alpha$ is an algebra homomorphism.

i) $\alpha: \operatorname{Orth}(X)^{\sim} \rightarrow \operatorname{Orth}\left(X^{\sim}\right), \quad \alpha(T) x^{\prime}=T \cdot x^{\prime}, \quad$ for $T \in \operatorname{Orth}(X)^{\sim}, x^{\prime} \in X^{\sim}$.

$$
\alpha(T) x^{\prime}=T \cdot x^{\prime}
$$

$\alpha$ is a linear mapping :

a) $\alpha(T+S)=\alpha(T)+\alpha(S) \quad$ we must show that $\alpha(T+S) x^{\prime}=\alpha(T) x^{\prime}+\alpha(S) x^{\prime} \quad$ is true for all $x \in X$.

$\left[\alpha(T+S) x^{\prime}\right] \cdot x=\left[(T+S) \cdot x^{\prime}\right] \cdot x=(T+S)\left(x \cdot x^{\prime}\right) \quad$ From third product we have

$\left(T . x^{\prime}\right) x=T\left(x . x^{\prime}\right) \quad$ and

$(T+S)\left(x \cdot x^{\prime}\right)=T\left(x \cdot x^{\prime}\right)+S\left(x \cdot x^{\prime}\right)$

$=\left(T \cdot x^{\prime}\right) x+\left(S \cdot x^{\prime}\right) x$

$=\left[\alpha(T) x^{\prime}\right] \cdot x+\left[\alpha(S) x^{\prime}\right] \cdot x$.

b) $\alpha(\lambda T)=\lambda \alpha(T) \quad$ we must show that $\alpha(\lambda T) x^{\prime}=\lambda \alpha(T) x^{\prime}$ is true for all $x \in X$.

$$
\begin{aligned}
\left(\left[\alpha(\lambda T) \cdot x^{\prime}\right]\right) \cdot x & =\left[(\lambda T) \cdot x^{\prime}\right] \cdot x, \quad x^{\prime} \in X^{\sim} \\
& =\lambda T\left(x \cdot x^{\prime}\right) \\
& =\lambda\left(T \cdot x^{\prime}\right) \cdot x \\
& =\lambda\left[\alpha(T) \cdot x^{\prime}\right] . x .
\end{aligned}
$$

ii) $\alpha$ is one-one [i.e. $T \neq 0 \Rightarrow \alpha(T) \neq 0$.]

$0 \neq T \in \operatorname{Orth}(X)^{\sim}$ there is a $\exists y \in \operatorname{Orth}(X)^{\sim}$

Let us take as $y=x . x^{\prime} \in \operatorname{Orth}(X)^{\sim}$

$T y \neq 0$

$T\left(x . x^{\prime}\right) \neq 0$

(T. $\left.x^{\prime}\right) \cdot x=0$

$\left[\alpha(T) x^{\prime}\right] x \neq 0 \Rightarrow \alpha(T) \neq 0$. 
iii) $\alpha$ is an algebra homomorphism, to prove this claim,

$$
\begin{aligned}
& \alpha(T . S)=\alpha(T) \alpha(S) \quad \text { for all } x \in X . \\
& \begin{aligned}
{\left[\alpha(T . S) . x^{\prime}\right] . x } & =(T: S)\left(x . x^{\prime}\right) \quad, \quad x . x^{\prime} \in \operatorname{Orth}(X)^{\sim} \\
& =T\left(S\left(x . x^{\prime}\right)\right) \\
& =T\left(\alpha(S) x^{\prime}\right) \cdot x \\
& =T\left(S . x^{\prime}\right) \cdot x \quad, \quad S . x^{\prime} \in X^{\sim} \\
& =\left[T\left(S . x^{\prime}\right)\right] . x \\
& =\alpha(T) \alpha(S) .
\end{aligned}
\end{aligned}
$$

Lemma 2[1,5] : Let $x \in X, \quad f \in X^{\sim}$.

If the mapping $f . x: \operatorname{Orth}(X) \rightarrow R$ is defined by $(f \cdot x)(\pi)=(f \circ \pi) \cdot x \quad$, for $\pi \in \operatorname{Orth}(X)$ then $f . x \in \operatorname{OrthX}^{\sim}$.

Proof : Consider the mapping (2)

$$
\begin{aligned}
& X \times X^{\sim} \rightarrow \text { Orth }^{\sim} \\
& (x, f) \rightarrow x \circ f \quad, \quad x \circ f \in \operatorname{Orth}^{\sim} \quad, \quad x \circ f \in \operatorname{Orth}(X) \rightarrow R \quad \text { Then, we obtain } \\
& (x . f)(\pi)=(f \circ \pi) . x . \\
& \text { Let } \beta: \text { Orth }(X) \rightarrow L_{b}(X) \text { be a mapping defined by } \\
& T \rightarrow \beta(T) x=T x .
\end{aligned}
$$

Next, we obtain the following equalities:

$$
\begin{aligned}
& \begin{aligned}
(x f)(T)= & (T x)(f) \quad, \quad f \in X^{\sim} \\
& =\beta(T) x
\end{aligned} \\
& (x . f)(\pi)=(\pi x)(f) \\
& =(\beta(\pi) x) . f \\
& =f(\beta(\pi) x) \\
& =f(\pi x) . \quad \text { So, } \\
& (x . f)(\pi)=f(\pi x) \text { and } \quad x f \in \text { OrthX }^{\sim} .
\end{aligned}
$$

Lemma 3 : Suppose the mapping $\phi: X^{\sim \sim} \rightarrow \operatorname{Orth}(X)^{\sim}$ is defined by 


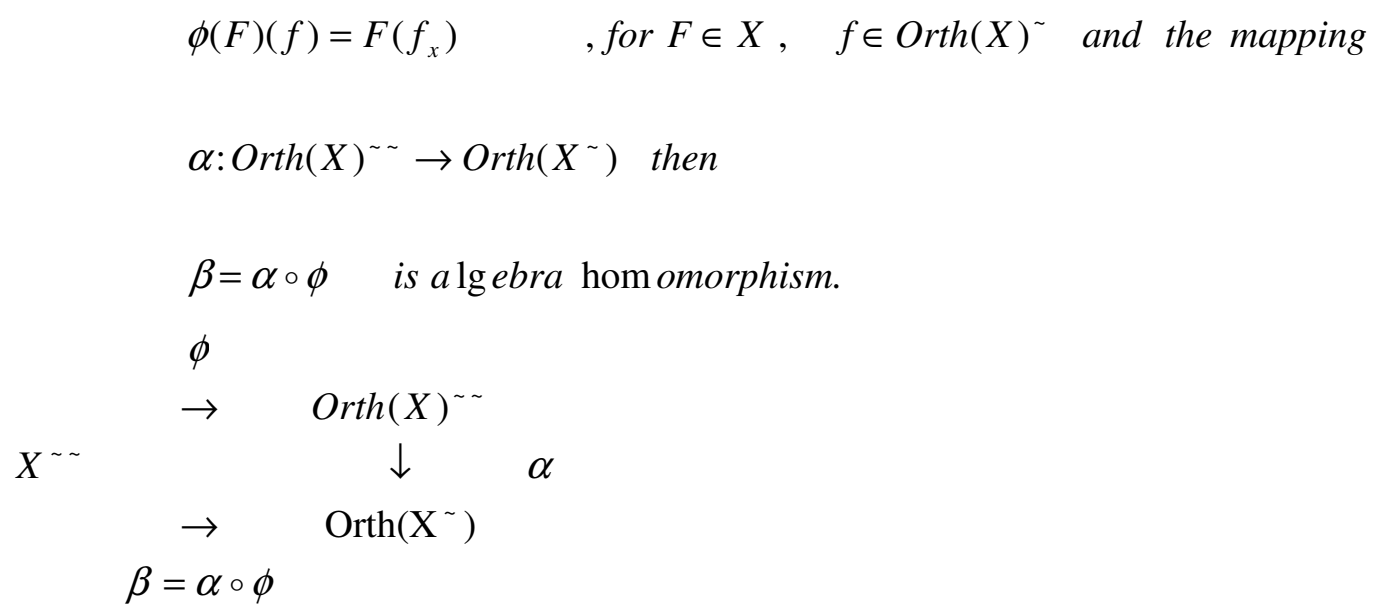

Proof :We must show

$$
\begin{aligned}
\beta(F . G) & =\beta(F) \cdot \beta(G) \quad \text { for } \quad F, G \in X^{\sim \sim} \\
\beta(F . G) & =(\alpha \circ \phi)(F . G) \\
& =\alpha(\phi(F . G)) \quad \text { we know } \phi \text { is hom omorphism }[1] \\
& =\alpha(\phi(F) \cdot \phi(G)) \\
& =\alpha\left(\phi(F) \cdot \alpha(\phi(G)) \quad\left(X^{\sim} \text { is } f-\bmod \text { ule over Orth }(X)^{\sim}\right)\right. \\
& =(\alpha \circ \phi)(F)(\alpha \circ \phi)(G) \\
& =\beta(F) \beta(G) .
\end{aligned}
$$

For every $f \in\left(X^{\sim}\right)^{+}$, defined $\varepsilon(f)$ to be the set of all extensions of $f$ in $\left(\operatorname{Orth}(X)^{\sim}\right)^{+}$, [1]. That is, $\mathcal{E}(f)=\left\{g \in \operatorname{Orth}(X)^{\sim}: 0 \leq g\right.$ and $\left.g_{x}=f\right\}$.

Proposition 4 : Let $f$ be an element of order dual $X^{\sim}$ of $X$ with $\varepsilon(f) \neq 0$. If $x \in X^{+}$, then $f . x=0$ implies $f(x)=0,[1]$.

Proof : Let us consider the set,

$\varepsilon(f)=\left\{g \in \operatorname{Orth}(X)^{\sim}: 0 \leq g\right.$ and $\left.g_{x}=f\right\}$. for $f \in X^{\sim}, x \in X$.

Let $\quad 0=f . x \in \operatorname{Orth}\left(X^{\sim}\right)$

$0(T)=f(T . x) \quad, \quad \forall T \in \operatorname{Orth}(X)$

$0=f(T x)$

If we take $T=I$ 
$f(I x)=0 \Rightarrow f(x)=0$.

\section{References}

[1] K. Boulabiar, J. Jamel,: The Order Bidual of f-algebras revisited,Positivity, 15,no.2, ( 2010 ), 271-279.

[2] C.D. Aliprantis, O. Burkinshaw: Positive Operators. Springer, Dordrecht ( 2006 ).

[3] R. Arens: The adjoint of bilinear operation. Proc. Am. Math. Soc. 2 ( 1951 ), 839848.

[4] S.J.Bernau, C.B. Huijsmans:The order bidual of almost f-algebras and d-algebras. Trans. Am. Math. Soc. 347 ( 1995 ), 4259-4275.

[5] A. Bigard, K. Keimel, S. Wolfenstein: Groups et Anneaux Reticules. Lecture Notes Math., vol. 608. Springer, Berlin ( 1977 ).

[6] C.B.Huijsmans : The order bidual of lattice-ordered algebras II. J. Oper. Theory 22, (1989 ), 277-290.

[7] C.B. Huijsmans : The order bidual of lattice-ordered algebras. J. Funct. Anal.59, (1984), 41-64.

Received: September, 2012 